\title{
Measuring glomerular filtration rate using ${ }^{51} \mathrm{Cr}$-EDTA: body surface area normalization before or after Bröchner-Mortensen correction?
}

\author{
Hans Pottel ${ }^{a}$, Liesbeth Hoste ${ }^{a}$, Liesbeth De Waele ${ }^{b, c}$, Elke Braat ${ }^{\text {d,f }}$, \\ Kristof Baete $^{\mathrm{e}, \mathrm{g}}$, Karolien Goffin ${ }^{\mathrm{e}, \mathrm{g}}$, Elena Levtchenko ${ }^{\mathrm{d}, \mathrm{f}}$ and Olivier Gheysens ${ }^{\mathrm{e}, \mathrm{g}}$
}

\begin{abstract}
Background Guidelines for measuring glomerular filtration rate (GFR) using ${ }^{51} \mathrm{Cr}$-EDTA require normalizing of GFR for body surface area (BSA) before applying the BröchnerMortensen (BM) correction. The guideline explicitly mentions the importance of performing BSA normalization before $\mathrm{BM}$ correction and that this is particularly important in children in whom the effects of BSA normalization are largest.
\end{abstract}

\begin{abstract}
Materials and methods We theoretically showed that the order of applying BM correction and BSA indexing is indeed important for patient populations having a low BSA and a high slow GFR. We then compared the exact GFR, obtained from the double-exponential concentration-time curve in Duchenne muscular dystrophy (DMD) patients, with the GFR obtained from the slow compartment method using the BM correction.

Results The median GFR for the 20 DMD patients obtained from the BSA-BM order deviates $5.40 \%$ from the exact GFR $(P=0.0006)$, whereas the median GFR obtained from the BM-BSA order deviates only $-0.05 \%(P>0.05)$ from the exact GFR, resulting in a median of differences of $5.50 \%$ between the two methods $(P<0.0001)$.
\end{abstract}

\section{Introduction}

Renal inulin clearance, performed by loading and continuously infusing inulin and collecting timed urine samples through a bladder catheter, is the gold standard for measuring glomerular filtration rate (GFR) [1]. As inulin is no longer readily available and this method requires extensive technical assistance, alternative methods using radioactive agents, such as ${ }^{51} \mathrm{Cr}$-EDTA, ${ }^{99} \mathrm{~m}$ Tc-diethylenetriaminepentaacetic acid, ${ }^{125} \mathrm{I}$ or ${ }^{131}$ I-iothalamate, are applied as surrogate markers of GFR. There are, however, problems with each of these agents: ${ }^{51} \mathrm{Cr}$-EDTA is not available in the USA; the renal handling of ${ }^{99 \mathrm{~m}} \mathrm{Tc}$-diethylenetriaminepentaacetic acid can vary depending on the commercial source, and ${ }^{131}$ I-iothalamate is readily secreted by the kidney [2]. In general, these agents are given by a single bolus injection and the clearance from the blood is measured through serial blood sampling. GFR is then calculated based on the area under the plasma clearance curve (AUC) of a

The work was performed in UZ Leuven Hospital, Belgium.
Conclusion The correct order of application in this DMD population should be BM correction first, followed by BSA indexing, and not vice versa. In general, the order of applying the BM correction and BSA normalization becomes more important with increasing slow GFR and extreme low BSA. The order of application is of less importance for people with normal BSA and/or normal GFR. Nucl Med Commun 00:000-000 @ 2014 Wolters Kluwer Health | Lippincott Williams \& Wilkins.

Nuclear Medicine Communications 2014, 00:000-000

Keywords: body surface area normalization, Bröchner-Mortensen correction, double-exponential decay curve, Duchenne muscular dystrophy patients, glomerular filtration rate, slow compartment method

Departments of a Public Health and Primary Care at Kulak, 'Development and Regeneration at Kulak, KU Leuven Kulak, Kortrijk, Departments of ${ }^{\mathrm{C} P a e d i a t r i c}$ Neurology, ${ }^{\mathrm{d}}$ Paediatric Nephrology, ${ }^{\mathrm{e}}$ Nuclear Medicine, University Hospital Leuven, Departments of ${ }^{\mathrm{f}}$ Development and Regeneration and Imaging and Pathology, KU Leuven, Leuven, Belgium

Correspondence to Hans Pottel, $\mathrm{PhD}$, Department of Public Health and Primary Care at Kulak, KU Leuven Kulak, Etienne Sabbelaan 53, B-8500 Kortrijk, Belgium Tel: + 3256 246022; fax: + 3256 246994;

e-mail: hans.pottel@kuleuven-kulak.be

Received 8 July 2014 Accepted 24 July 2014

mathematical fit through the data points. Several methods can be applied to obtain the clearance curve. The rate of tracer clearance can be best described by a doubleexponential decay curve, leading to the most accurate determination of both the fast and the slow decaying part. This double-exponential decay curve, however, requires up to nine blood samples, which makes it less convenient in daily clinical practice. Therefore, alternative simplified sampling strategies have been proposed to determine the slow decay compartment only. This one-compartment clearance is fitted with a monoexponential decay function and allows the calculation of the so-called slow GFR. This slow GFR overestimates the true GFR, because the early fast - but missing - compartment cannot be integrated, leading to the underestimation of the AUC. The overestimation is small (even negligible) for low renal function but increases nonlinearly with higher and normal renal function. The overestimation of the true GFR, or, more exactly, the underestimation of the AUC, can be corrected using the Bröchner-Mortensen (BM) formula, or with variants of that correction [3-5].

DOI: 10.1097/MNM.0000000000000186 
In the original 1972 publication of BM [3], the slow GFR is corrected using a polynomial of the second degree:

$$
\begin{aligned}
\text { Exact GFR }= & 0.990778 \times(\text { slow GFR }) \\
& -0.001218 \times(\text { slow GFR })^{2},
\end{aligned}
$$

where both exact GFR and slow GFR are expressed in $\mathrm{ml} / \mathrm{min}$.

As GFR is usually indexed or normalized by the body surface area (BSA), this exact GFR is further adjusted as follows:

$$
\text { Exact } \mathrm{GFR}_{\mathrm{BSA}}=\frac{\text { Exact GFR } \times 1.73}{\mathrm{BSA}},
$$

where the BSA is usually calculated with the Du Bois and Du Bois formula [6].

$$
\text { BSA }=0.007184 \times \text { height } t^{0.725} \times \text { weight }^{0.425} .
$$

BSA is expressed in $\mathrm{m}^{2}$, so finally the exact $\mathrm{GFR}_{\mathrm{BSA}}$ is expressed in $\mathrm{ml} / \mathrm{min} / 1.73 \mathrm{~m}^{2}$.

As in the original BM publication of 1972 BSA normalization is not even mentioned, correction for BM should be done first, followed by BSA normalization.

Guidelines for measuring GFR using plasma sampling [4], adopted by the British Nuclear Medicine Society, normalize GFR for BSA first before applying the BM correction. The guideline mentions explicitly that it is important to perform the BSA and AUC (or BM) corrections in the right order and that this is particularly important for children in whom the effects of BSA normalization have the largest consequences. The basis for this statement is unclear, as no reference is cited in the text.

In a recent technical review, Murray et al. [5] suggested that BSA normalization be performed before applying the quadratic BM correction using only a one-compartment decay, without any scientific argumentation, but with reference to Picciotto et al. [7]. Furthermore, Murray and colleagues state that the BM correction is known to overestimate GFR for patients with a renal clearance of more than $80 \mathrm{ml} / \mathrm{min} / 1.73 \mathrm{~m}^{2}$, particularly for patients with a BSA below $1 \mathrm{~m}^{2}$, which is the case in paediatric patients. After careful reading of the article by Picciotto et al. [7] we could not discover the basis for his statement.

Therefore, through this study we aim to determine which step comes first: BSA normalization or AUC correction. We performed a theoretical analysis assessing the effect on exact GFR calculated from the slow GFR when applying the BM correction first followed by BSA normalization compared with BSA normalization first and then $\mathrm{BM}$ correction. We also investigated whether and under which circumstances these differences became clinically important and we used patient data to determine the correct order of application.

\section{Materials and methods}

${ }^{51} \mathrm{Cr}$-EDTA procedures were performed in 20 patients aged between 5 and 22 years with Duchenne muscular dystrophy (DMD). The study was performed in accordance with the ethical standards laid down in the 1964 Declaration of Helsinki and its later amendments. Consent forms were signed by parents of participants below 17 years of age or by participants older than 18 years of age. Assent forms were signed by participants of 5-17 years of age.

From each patient, eight blood samples were collected at fixed time points (15-30-45-60-120-180-240-300 min after intravenous bolus injection of ${ }^{51} \mathrm{Cr}$-EDTA) and the concentration-time curve was fitted by a doubleexponential decay:

$$
c(t)=A \mathrm{e}^{-\alpha t}+B \mathrm{e}^{-\beta t} .
$$

The exact GFR was calculated from this curve as follows:

$$
\begin{aligned}
\text { Exact GFR } & =\frac{\text { Administered activity }}{\text { AUC }} \\
& =\frac{\text { Administered activity }}{\frac{A}{\alpha}+\frac{B}{\beta}} .
\end{aligned}
$$

The slow GFR was obtained by fitting the concentration values at 2, 3, 4 and $5 \mathrm{~h}$ after injection, as described in [4], with a monoexponential decay curve:

$$
c(t)=M \mathrm{e}^{-\gamma t},
$$

$$
\begin{aligned}
\text { Slow GFR } & =\frac{\text { Administered activity }}{\text { AUC }} \\
& =\frac{\text { Administered activity }}{\frac{M}{\gamma}} .
\end{aligned}
$$

This slow GFR was then corrected in two ways.

(1) BM correction first, followed by BSA normalization: $\mathrm{GFR}_{\mathrm{BM}}=$

$$
\begin{gathered}
0.990778 \times(\text { slow GFR })-0.001218 \times(\text { slow GFR })^{2}, \\
\text { GFR }_{\mathrm{BM}-\mathrm{BSA}}=\frac{\mathrm{GFR}_{\mathrm{BM}} \times 1.73}{\mathrm{BSA}} .
\end{gathered}
$$

(2) BSA normalization, followed by BM correction: $\mathrm{GFR}_{\mathrm{BSA}}=\frac{\text { Slow GFR } \times 1.73}{\mathrm{BSA}}$,

$$
\begin{aligned}
\operatorname{GFR}_{\mathrm{BSA}-\mathrm{BM}}= & 0.990778 \times\left(\mathrm{GFR}_{\mathrm{BSA}}\right) \\
& -0.001218 \times\left(\mathrm{GFR}_{\mathrm{BSA}}\right)^{2} .
\end{aligned}
$$

All statistical analyses, including nonlinear curve fitting, were performed with GraphPad Prism 6.0 (GraphPad 
Software Inc., La Jolla, California, USA). Paired differences were tested against zero with the Wilcoxon signedrank test.

\section{Results}

\section{Theoretical analysis}

Mathematically, the difference between the results obtained with both orders of application can be calculated as follows:

$$
\begin{gathered}
\Delta=\mathrm{GFR}_{\mathrm{BM}-\mathrm{BSA}}-\mathrm{GFR}_{\mathrm{BSA}-\mathrm{BM}}, \\
\Delta=\left[0.990778 \times(\text { slow } \mathrm{GFR})-0.001218 \times(\text { slow } \mathrm{GFR})^{2}\right] \\
\times \frac{1.73}{\mathrm{BSA}}-0.990778 \times\left(\text { slow } \mathrm{GFR} \times \frac{1.73}{\mathrm{BSA}}\right)+0.001218 \\
\times\left(\text { slow } \mathrm{GFR} \times \frac{1.73}{\mathrm{BSA}}\right)^{2} .
\end{gathered}
$$

The linear component is cancelled out, leaving us with the following quadratic terms:

$$
\begin{aligned}
\Delta= & -0.001218 \times(\text { slow GFR })^{2} \times \frac{1.73}{\mathrm{BSA}}+0.001218 \\
& \times(\text { slow GFR })^{2} \times\left(\frac{1.73}{\mathrm{BSA}}\right)^{2},
\end{aligned}
$$

$\Delta=-0.001218 \times(\text { slow GFR })^{2} \times\left(\frac{1.73}{\mathrm{BSA}}\right) \times[1-1.73 / \mathrm{BSA}]$

The relative difference $\Delta /\left[\left(G R_{\mathrm{BM}-\mathrm{BSA}}+\mathrm{GFR}_{\mathrm{BSA}-}\right.\right.$ $\left.{ }_{B M}\right) / 2$ ] in terms of BSA and slow GFR is presented in Table 1. This difference is zero when $\mathrm{BSA}=1.73 \mathrm{~m}^{2}$, independent of the slow GFR value. For $1.4 \leq \mathrm{BSA}$ $\leq 2.3 \mathrm{~m}^{2}$ and slow $\mathrm{GFR}<140 \mathrm{ml} / \mathrm{min}$, the absolute differences between both approaches are smaller than $5 \%$ (bold italic in Table 1), which could be considered clinically insignificant but become significant $(>10 \%)$ when BSA is below $1.4 \mathrm{~m}^{2}$ and with increasing slow GFR (italic in Table 1). In patients with very low BSA $\left(\mathrm{BSA}<1 \mathrm{~m}^{2}\right)$, the differences become very large, especially at high slow GFR (slow GFR $>100 \mathrm{ml} / \mathrm{min}$ ). The BM-BSA application order may thus have a large effect for children and adults with extreme low BSA, especially for high GFR values.

For example, an individual with $\mathrm{BSA}=0.6 \mathrm{~m}^{2}$ (average BSA of a 3-year-old child, with average height of $95 \mathrm{~cm}$ and weight of $14 \mathrm{~kg}$ ) and slow $\mathrm{GFR}=50 \mathrm{ml} / \mathrm{min}$ has a true GFR of $134.1 \mathrm{ml} / \mathrm{min} / 1.73 \mathrm{~m}^{2}$ when the order BMBSA is applied and $117.5 \mathrm{ml} / \mathrm{min} / 1.73 \mathrm{~m}^{2}$ when BSA-BM is applied, resulting in an absolute difference of $16.6 \mathrm{ml} /$ $\mathrm{min} / 1.73 \mathrm{~m}^{2}$, or a relative difference of $16.6 /$ $[(134.1+117.5) / 2]=13.1 \%$.

To give another example, an individual with $\mathrm{BSA}=0.6 \mathrm{~m}^{2}$ and slow $\mathrm{GFR}=100 \mathrm{ml} / \mathrm{min}$ has a true GFR of $251 \mathrm{ml} / \mathrm{min} / 1.73 \mathrm{~m}^{2}$ when the order BM-BSA is applied and $184 \mathrm{ml} / \mathrm{min} / 1.73 \mathrm{~m}^{2}$ when BSA-BM is used, an absolute difference of $67 \mathrm{ml} / \mathrm{min} / 1.73 \mathrm{~m}^{2}$, or a relative difference of $67 / 217.5=30.4 \%$.

\section{Glomerular filtration rate measurement in patients with Duchenne muscular dystrophy}

The calculations in the previous section illustrate that there are significant differences between both methods, and that it is therefore important to apply the correct order, especially in patients with low BSA and high slow GFR. The question still remains as to which order is correct and should be applied in those patients. DMD patients are a subgroup of children that reflects this

\begin{tabular}{|c|c|c|c|c|c|c|c|c|c|c|c|c|c|c|c|c|c|c|c|c|}
\hline & \multicolumn{20}{|c|}{$\mathrm{BSA}\left(\mathrm{m}^{2}\right)$} \\
\hline & 0.5 & 0.6 & 0.7 & 0.8 & 0.9 & 1 & 1.1 & 1.2 & 1.3 & 1.4 & 1.5 & 1.6 & 1.73 & 1.8 & 1.9 & 2.0 & 2.1 & 2.2 & 2.3 & 2.4 \\
\hline \multicolumn{21}{|c|}{ Slow GFR (ml/min) } \\
\hline 10 & 3.1 & 2.4 & 1.8 & 1.5 & 1.2 & 0.9 & 0.7 & 0.6 & 0.4 & 0.3 & 0.2 & 0.1 & 0.0 & 0.0 & -0.1 & -0.2 & -0.2 & -0.3 & -0.3 & -0.3 \\
\hline 20 & 6.4 & 4.9 & 3.8 & 3.0 & 2.4 & 1.9 & 1.5 & 1.1 & 0.8 & 0.6 & 0.4 & 0.2 & 0.0 & -0.1 & -0.2 & -0.3 & -0.4 & -0.5 & -0.6 & -0.7 \\
\hline 30 & 9.9 & 7.5 & 5.8 & 4.6 & 3.6 & 2.8 & 2.2 & 1.7 & 1.3 & 0.9 & 0.6 & 0.3 & 0.0 & -0.1 & -0.3 & -0.5 & -0.7 & -0.8 & -0.9 & -1.1 \\
\hline 40 & 13.6 & 10.2 & 7.9 & 6.2 & 4.9 & 3.8 & 3.0 & 2.3 & 1.7 & 1.2 & 0.8 & 0.4 & 0.0 & -0.2 & -0.5 & -0.7 & -0.9 & -1.1 & -1.3 & -1.4 \\
\hline 50 & 17.5 & 13.1 & 10.1 & 7.9 & 6.2 & 4.9 & 3.8 & 2.9 & 2.2 & 1.6 & 1.0 & 0.5 & 0.0 & -0.3 & -0.6 & -0.9 & -1.1 & -1.4 & -1.6 & -1.8 \\
\hline 60 & 21.7 & 16.2 & 12.4 & 9.7 & 7.6 & 6.0 & 4.7 & 3.6 & 2.7 & 1.9 & 1.2 & 0.6 & 0.0 & -0.3 & -0.7 & -1.1 & -1.4 & -1.7 & -2.0 & -2.2 \\
\hline 70 & 26.2 & 19.5 & 14.9 & 11.6 & 9.1 & 7.1 & 5.5 & 4.2 & 3.2 & 2.2 & 1.5 & 0.8 & 0.0 & -0.4 & -0.8 & -1.3 & -1.6 & -2.0 & -2.3 & -2.6 \\
\hline 80 & 31.0 & 22.9 & 17.4 & 13.5 & 10.6 & 8.3 & 6.4 & 4.9 & 3.7 & 2.6 & 1.7 & 0.9 & 0.0 & -0.4 & -1.0 & -1.5 & -1.9 & -2.3 & -2.7 & -3.0 \\
\hline 90 & 36.1 & 26.5 & 20.1 & 15.6 & 12.2 & 9.5 & 7.4 & 5.6 & 4.2 & 3.0 & 1.9 & 1.0 & 0.0 & -0.5 & -1.1 & -1.7 & -2.2 & -2.6 & -3.0 & -3.4 \\
\hline 100 & 41.7 & 30.4 & 23.0 & 17.7 & 13.8 & 10.8 & 8.4 & 6.4 & 4.7 & 3.4 & 2.2 & 1.1 & 0.0 & -0.5 & -1.2 & -1.9 & -2.4 & -3.0 & -3.4 & -3.8 \\
\hline 110 & 47.6 & 34.5 & 26.0 & 20.0 & 15.5 & 12.1 & 9.4 & 7.2 & 5.3 & 3.8 & 2.4 & 1.3 & 0.0 & -0.6 & -1.4 & -2.1 & -2.7 & -3.3 & -3.8 & -4.3 \\
\hline 120 & 54.1 & 38.9 & 29.2 & 22.4 & 17.3 & 13.5 & 10.4 & 7.9 & 5.9 & 4.2 & 2.7 & 1.4 & 0.0 & -0.7 & -1.5 & -2.3 & -3.0 & -3.6 & -4.2 & -4.7 \\
\hline 130 & 61.1 & 43.6 & 32.5 & 24.9 & 19.2 & 14.9 & 11.5 & 8.8 & 6.5 & 4.6 & 3.0 & 1.6 & 0.0 & -0.7 & -1.7 & -2.5 & -3.3 & -4.0 & -4.6 & -5.2 \\
\hline 140 & 68.7 & 48.7 & 36.1 & 27.5 & 21.2 & 16.4 & 12.7 & 9.6 & 7.1 & 5.0 & 3.2 & 1.7 & 0.0 & -0.8 & -1.8 & -2.8 & -3.6 & -4.3 & -5.0 & -5.6 \\
\hline 150 & 77.0 & 54.1 & 39.9 & 30.3 & 23.3 & 18.0 & 13.8 & 10.5 & 7.8 & 5.5 & 3.5 & 1.9 & 0.0 & -0.9 & -2.0 & -3.0 & -3.9 & -4.7 & -5.5 & -6.1 \\
\hline
\end{tabular}
extreme situation very well (Table 2). We used their GFR data to determine the correct order of applying the $\mathrm{BM}$ correction and BSA normalization.

Table 1 Relative difference $\% \Delta /\left[\left(\mathrm{GFR}_{\mathrm{BM}-\mathrm{BSA}}+\mathrm{GFR}_{\mathrm{BSA}-\mathrm{BM}}\right) / 2\right]$, for different values of slow glomerular filtration rate $(\mathrm{ml} / \mathrm{min})$ and body surface areas $\left(\mathrm{m}^{2}\right)$

Italic, |difference| $\geq 10 \%$; bold roman, |difference| $\geq 5 \%$ and $<10 \%$; bold italic, |difference $\mid<5 \%$.

BSA, body surface area; GFR, glomerular filtration rate. 
Table 2 Characteristics of 20 Duchenne muscular dystrophy patients

\begin{tabular}{lcc}
\hline & Mean (SD) & Range \\
\hline Age (years) & $14.7(4.0)$ & $5-22$ \\
BSA $\left(\mathrm{m}^{2}\right)$ & $1.33(0.27)$ & $0.74-1.75$ \\
Exact GFR $\left(\mathrm{ml} / \mathrm{min} / 1.73 \mathrm{~m}^{2}\right)$ & $137.0(29.1)$ & $85.7-226.3$ \\
GFR & $138.1(34.3)$ & $75.8-236.7$ \\
GFR & $127.9(23.4)$ & $75.8-181.9$ \\
\hline
\end{tabular}

BM, Bröchner-Mortensen; BSA, body surface area; GFR, glomerular filtration rate.

Fig. 1

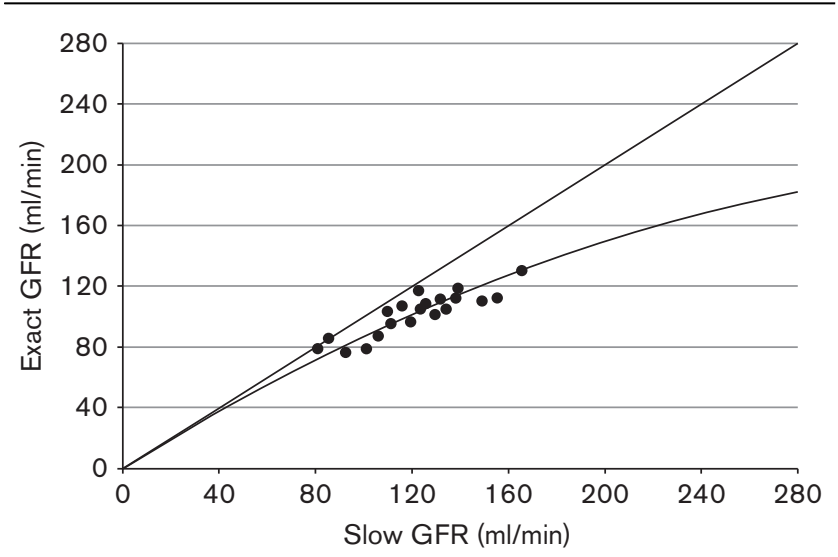

Exact GFR and slow GFR ( $\mathrm{ml} / \mathrm{min}$ ) for 20 DMD patients (circles) together with the identity line (straight line) and the BM correction line (curved line, $0.990778 \times$ slow GFR-0.001218 $\times$ slow GFR ${ }^{2}$ ). BM, Bröchner-Mortensen; DMD, Duchenne muscular dystrophy; GFR, glomerular filtration rate.

Figure 1 displays exact GFR and slow GFR values of 20 DMD patients before BSA normalization and illustrates that the GFR values of DMD patients are close to the $\mathrm{BM}$ correction line, proving the validity of the BM correction. Figure 2 shows exact GFR and slow GFR values, normalized to BSA. Applying BSA normalization first in this population results in higher values of both slow and exact GFR, extending the range of GFR values from $160 \mathrm{ml} / \mathrm{min}$ in Fig. 1 to $280 \mathrm{ml} / \mathrm{min} / 1.73 \mathrm{~m}^{2}$ in Fig. 2 . Figure 2 also shows a larger offset from the BM correction line, as compared with Fig. 1.

The relative differences between GFR values, calculated from the slow GFR for both application orders (BM-BSA vs. BSA-BM), and exact GFR values are presented in Fig. 3. The median of paired differences between both methods is $5.50 \%$ $(P<0.0001)$. The median GFR calculated from the BM-BSA order deviated only $-0.05 \%$ from the double-exponentially calculated exact GFR $(P>0.05)$, and a deviation of $5.40 \%$ was found between the exact GFR and the GFR obtained from the BSA-BM application order $(P=0.0006)$.

\section{Discussion}

GFR can be determined accurately by measuring the rate of radiotracer clearance from blood using a simplified
Fig. 2

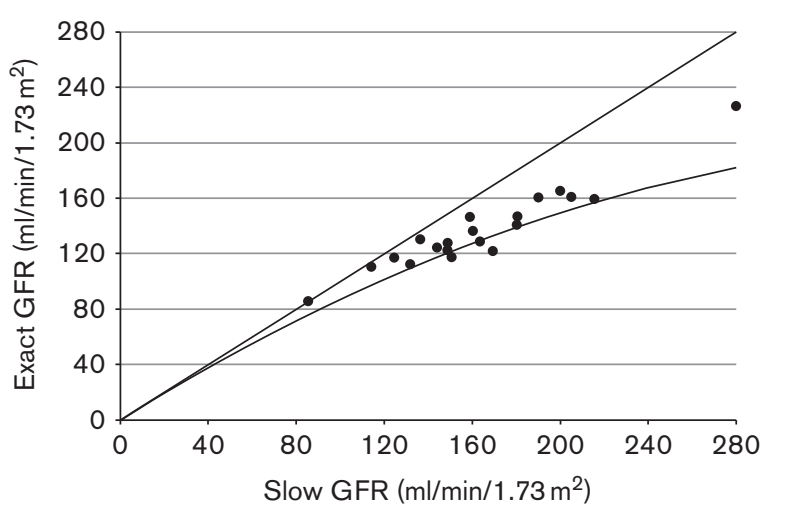

Exact GFR and slow GFR normalized for BSA $\left(\mathrm{ml} / \mathrm{min} / 1.73 \mathrm{~m}^{2}\right)$ for 20 DMD patients, together with the identity line (straight line) and the BM correction line (curved line, $0.990778 \times$ slow GFR $-0.001218 \times$ slow $\mathrm{GFR}^{2}$ ). BM, Bröchner-Mortensen; BSA, body surface area; DMD, Duchenne muscular dystrophy; GFR, glomerular filtration rate.

Fig. 3

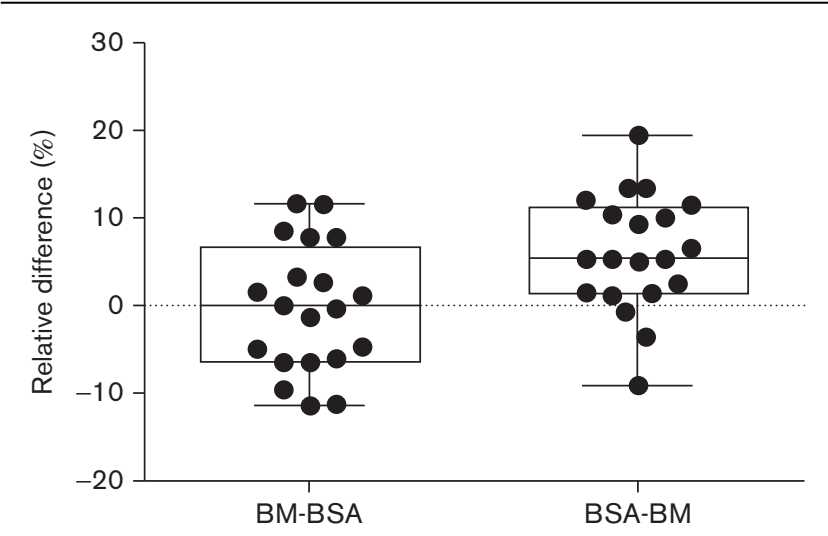

The relative differences (a positive difference corresponds to an underestimation) from the exact GFR for the GFR calculated from the slow GFR according to two application orders: BM-BSA (first BM, followed by BSA) and BSA-BM (first BSA, followed by BM) for the 20 DMD patients. The zero line corresponds to the exact GFR, as obtained from the double-exponential decay. The median of differences is $5.50 \%$ $(P<0.0001$, Wilcoxon signed-rank test). BM, Bröchner-Mortensen; BSA, body surface area; DMD, Duchenne muscular dystrophy; GFR, glomerular filtration rate.

sampling strategy that is convenient in daily clinical practice, given that the necessary corrections are applied in the right order. The British guidelines [4] for measuring GFR recommends that the BM equation can be used but 'if the BM correction is used then it is important that the BSA and AUC corrections are applied in the right order. This is particularly important for results in children when the effects of the BSA correction are greatest'. According to these guidelines, BSA indexing should be applied first, followed by BM correction. However, in this study in DMD patients, we showed that this order should be reversed and that the correct order of application is $\mathrm{BM}$ correction before BSA normalization. 
Apart from our data, other justifications for the reversal of the order can be found in the literature. First, the original BM article [3] mentions slow and exact GFR expressed in $\mathrm{ml} / \mathrm{min}$. BSA indexing is not discussed in this article. Second, the original BM correction formula $(0.990778 \times$ slow GFR $-0.001218 \times$ slow $G^{2} R^{2}$ ) [3] is built with data ranging from 10 to $170 \mathrm{ml} / \mathrm{min}$ for slow GFR, resulting in exact GFR values ranging between 10 and $140 \mathrm{ml} / \mathrm{min}$. By first applying BSA indexing for extremely low BSA values, the indexed slow GFR is out of the original range, requiring extrapolation, a common statistical mistake. There is no reason to assume that the relationship would continue much beyond the range of the data. Predictions substantially beyond that range are likely to be very wrong. Jødal and Brøchner-Mortensen [8] state that one cannot obtain clearance values higher than about $200 \mathrm{ml} / \mathrm{min}$ using the BM correction formula. They further mention that on using second-order polynomials the reference clearance is increasingly underestimated at high clearance values (on average $10 \%$ at $175 \mathrm{ml} / \mathrm{min} / 1.73 \mathrm{~m}^{2}$ ) [9]. However, it should be noted that the maximum value of $200 \mathrm{ml} / \mathrm{min}$ corresponds with a slow GFR of $406.7 \mathrm{ml} / \mathrm{min}$ $[=0.990778 /(2 \times 0.001218)]$, a value that is more than double the range of the original $\mathrm{BM}$ correction formula, and a value so high that it has never been reported before. Further, the value of $175 \mathrm{ml} / \mathrm{min} / 1.73 \mathrm{~m}^{2}$ is outside the range of the original $\mathrm{BM}$ correction formula. Application of the BM correction formula should be restricted to the range $0-170 \mathrm{ml} / \mathrm{min}$ for the slow GFR. This corresponds to a range for the true GFR of $0-133 \mathrm{ml} / \mathrm{min}$. When the slow GFR is out of range, there is no other possibility than obtaining the complete concentration-time decay, including the fast component. One should never rely on extrapolation. In Fig. 1, we illustrate that the BM correction is still applicable for DMD patients when applied on the slow GFR expressed in $\mathrm{ml} / \mathrm{min}$, but the deviation from the BM equation becomes large (Fig. 2) when applied to the BSAindexed slow GFR. As all slow GFR values for our DMD population were still within the original range of the BM equation (largest slow GFR was $154 \mathrm{ml} / \mathrm{min}$ ), application of this correction formula does not require extrapolation, only interpolation. When applied to the BSA-normalized values, five of 20 patients had BSA-normalized slow GFR values larger than $170 \mathrm{ml} / \mathrm{min} / 1.73 \mathrm{~m}^{2}$ (the largest slow GFR was $280 \mathrm{ml} / \mathrm{min} / 1.73 \mathrm{~m}^{2}$ ), requiring dangerous extrapolation.

The nonradioactive agent iohexol has become more standard for direct GFR measurements, because it is readily available, accepted in the USA, and not secreted, metabolized or reabsorbed by the kidney. Schwartz et al. [2] presented a BM-like correction formula when determining the slow GFR from the iohexol measurement, being of the same quadratic form with rather equivalent coefficients. However, Schwartz and colleagues applied BSA-normalized exact and slow GFR values during the construction of his correction equation, thus extending the range of application of his correction formula. In his equation, the order BSA-BM can be applied. Again, however, the correction formula should be applied within the original range of the data. It should be noted that, in his study, a median slow GFR of $60.83 \mathrm{ml} / \mathrm{min}$, with a third quartile of $97.53 \mathrm{ml} / \mathrm{min}$, and a median BSA of $1.66 \mathrm{~m}^{2}$, with a first quartile of $1.31 \mathrm{~m}^{2}$ were reported. As shown in Table 1 , a slow GFR of $100 \mathrm{ml} / \mathrm{min}$ would require a BSA less than $1.1 \mathrm{~m}^{2}$ to see a difference of $10 \%$ or more between both orders of BM/BSA application.

Piepsz and Ham [10] and Murray et al. [5] have previously warned about using BM correction for high clearance values because this correction underestimates GFR values greater than $100 \mathrm{ml} / \mathrm{min} / 1.73 \mathrm{~m}^{2}$ and introduces considerable compression of the clearance values higher than $140 \mathrm{ml} / \mathrm{min} / 1.73 \mathrm{~m}^{2}$. Monitoring of hyperfiltration will therefore be seriously hampered by using this correction factor. Piepsz, however, uses GFR values expressed in $\mathrm{ml} / \mathrm{min} / 1.73 \mathrm{~m}^{2}$ and not in $\mathrm{ml} / \mathrm{min}$ and refers to the article of BM published in 1974 [11], not to the original article of 1972 [3]. As mentioned before, in the original article of 1972, the BM correction formula is presented for slow and true GFR in $\mathrm{ml} / \mathrm{min}$, but in 1974 BM applied his original formula for BSA-normalized GFR, expressed in $\mathrm{ml} / \mathrm{min} / 1.73 \mathrm{~m}^{2}$. Especially for children with small BSA, the consequence of normalizing first is that the slow GFR (expressed in $\mathrm{ml} / \mathrm{min} / 1.73 \mathrm{~m}^{2}$ ) is outside the application range. From our study, we can conclude that the underestimation of the exact GFR is larger when BSA-BM is applied (median $= \pm 5 \%$ ), compared with application of BM-BSA (median $= \pm 0 \%$ ) (Fig. 3). In the article of 1974, BM [11] reported performing BSA indexing for slow GFR first followed by a correction to compensate for the underestimation of the AUC in 30 children suffering from nephrourological disorders, and this might be the basis for the current BSA$\mathrm{BM}$ order recommendation. In that article, the equation GFR $=1.01 \times$ slow GFR $-0.0017 \times(\text { slow } G F R)^{2}$ has been presented for children, where exact and slow GFR values are both expressed in $\mathrm{ml} / \mathrm{min} / 1.73 \mathrm{~m}^{2}$. However, the range of application in his study extended from 0 to $160 \mathrm{ml} / \mathrm{min} / 1.73 \mathrm{~m}^{2}$, which is much smaller than what we need here for our DMD patients (Fig. 2). The children in the 1974 article of BM all had GFR values lower than $120 \mathrm{ml} / \mathrm{min} / 1.73 \mathrm{~m}^{2}$, and consequently the difference between both application orders (BM-BSA or BSA-BM) is small (usually smaller than $5 \%$ ) in this population.

It is clear from our present study that the order of applying BM correction and BSA indexing is important for extreme patient populations having a low BSA and a high slow GFR, which is valid for DMD patients. The correct order of application in this population should be BM correction first followed by BSA normalization, and not vice versa. The order of application is of less importance for people with normal BSA $\left(>1.4 \mathrm{~m}^{2}\right)$ and/or normal GFR, where the difference will be mostly less than $5 \%$, which is clinically acceptable. 


\section{Acknowledgements}

\section{Conflicts of interest}

There are no conflicts of interest.

\section{References}

1 Arant BS Jr, Edelmann CM Jr, Spitzer A. The congruence of creatinine and inulin clearances in children: use of the Technicon AutoAnalyzer. $J$ Pediatr 1972; 81:559-561.

2 Schwartz GJ, Furth S, Cole SR, Warady B, Muñoz A. Glomerular filtration rate via plasma iohexol disappearance: pilot study for chronic kidney disease in children. Kidney Int 2006; 69:2070-2077.

3 Bröchner-Mortensen J. A simple method for the determination of glomerular filtration rate. Scand J Clin Lab Invest 1972; 30:271-274.

4 Fleming JS, Zivanovic MA, Blake GM, Burniston M, Cosgriff PS, British Nuclear Medicine Society. Guidelines for the measurement of glomerular filtration rate using plasma sampling. Nucl Med Commun 2004; 25:759-769.

5 Murray AW, Barnfield MC, Waller ML, Telford T, Peters AM. Assessment of glomerular filtration rate measurement with plasma sampling: a technical review. J Nucl Med Technol 2013; 41:67-75.
6 Du Bois D, Du Bois EF. A formula to estimate the approximate surface area if height and weight be known. Arch Intern Med 1916; 17:863-871.

7 Picciotto G, Cacace G, Cesana P, Mosso R, Ropolo R, De Filippi PG. Estimation of chromium-51 ethylene diamine tetra-acetic acid plasma clearance: a comparative assessment of simplified techniques. Eur J Nucl Med 1992; 19:30-35.

8 Jødal L, Brøchner-Mortensen J. Reassessment of a classical single injection ${ }^{51} \mathrm{Cr}$-EDTA clearance method for determination of renal function in children and adults. Part I: analytically correct relationship between total and one-pool clearance. Scand J Clin Lab Invest 2009; 69:305-313.

9 Brøchner-Mortensen J, Jødal L. Reassessment of a classical single injection ${ }^{51} \mathrm{Cr}$-EDTA clearance method for determination of renal function in children and adults. Part II: empirically determined relationships between total and one-pool clearance. Scand J Clin Lab Invest 2009; 69:314-322.

10 Piepsz A, Ham HR. Pediatric applications of renal nuclear medicine. Semin Nucl Med 2006; 36:16-35.

11 Bröchner-Mortensen J, Haahr J, Christoffersen J. A simple method for accurate assessment of the glomerular filtration rate in children. Scand J Clin Lab Invest 1974; 33:140-143. 